\title{
A Statistical Model of the Sleep-Wake Dynamics of the Cardiac Rhythm
}

\author{
PE McSharry ${ }^{1,2}$, GD Clifford $^{1}$ \\ ${ }^{1}$ Department of Engineering Science, University of Oxford, Oxford, UK \\ ${ }^{2}$ Mathematical Institute, University of Oxford, Oxford, UK \\ ${ }^{3}$ Harvard-MIT Division of Health Sciences \& Technology \\ Cambridge MA 02142, US
}

\begin{abstract}
The beat-to-beat heart rate varies considerably over the course of 24 hours. The Computers in Cardiology Challenge of 2002 was based on understanding and modelling the dynamics of the source of this variation. The diverse range of entries for simulating the $R R$ tachogram emphasises the level of ambiguity that exists when attempting to model the cardiovascular system over long time scales. In 2002, a model that combined both deterministic and stochastic descriptions of the data generating process was developed to reproduce both short term variations and long range correlations in RR intervals. In this paper these ideas are extended and new observations of sleep-wake transitions observed during sleep are incorporated. The new model more faithfully represents some of the key empirical characteristics that have been observed in actual recordings of healthy human subjects from the Physionet database. These characteristics include the distribution of $R R$ intervals during wake and sleep periods. In particular the model emulates the existence of a scale-free power law distribution during wakefulness and the exponential distribution (with a characteristic time scale) of sleep-wake activity during prolonged sleep. Concurrence with real data is demonstrated using detrended fluctuation analysis and multiscale entropy. In order to increase the model's utility for assessing biomedical signal processing methods applied to the RR interval time series, a label for each beat is available as either normal, ectopic or artifactual. Furthermore, a state label (wake or sleep) is available.
\end{abstract}

\section{Introduction}

The cardiac rhythm fluctuates throughout the day, responding to different demands on the body and varying levels of physical and mental activity. The ability of the heart to adapt to internal and external perturbations may provide useful information about its state of health. In particular, the loss of adaptability resulting from ageing or the onset of disease may in some case be detected from long recordings of cardiac inter-beat time intervals derived from the electrocardiogram [1].

Quantifying the self-similarity of such cardiac signals provides a means of distinguishing between health and disease and monitoring the effect of ageing $[2,3]$. One approach for measuring the self-similarity of a signal is to calculate its power-spectral density, $S(f)$, and estimate the power-law scaling exponent, $\beta$, that satisfies $S(f) \sim f^{-\beta}$. This exponent, $\beta$, may be viewed as a measure of the roughness of the time series, with smoother self-similar time series having larger values of $\beta$. For cardiac signals, a $1 / f$-like noise $(\beta=1)$ is found [4]. This value is midway between the complete randomness of white noise $(\beta=0)$ and the much smoother Brownian noise $(\beta=2)$ [5].

During sleep the human body is less exposed to physical activity and variations in the heartrate mainly result from neuronal activity. For this reason, it is advantageous to investigate the statistical properties of cardiac activity during sleep and during sleep-wake transitions. The scaling exponents have been found to vary between wake and sleep and also during different sleep stages $[1,6]$.

\section{Methods}

In this section, a method is described for producing realistic RR interval tachograms spanning twenty-four hours. These tachograms incorporate both sleep and wake activity. A dynamical model is used to govern the switching between states of sleep and wake [7]. Data simulated from a white noise process is transformed to produce segments of RR tachogram with pre-specified temporal and spectral properties [8].

\subsection{Sleep-wake dynamics}

Lo et al. [7] analysed the distribution of time durations spent in periods of sleep and wake for 39 full-night sleep records of 20 healthy subjects. These included 11 females 
and 9 males aged between 23 and 57 with an average sleep duration of 7.0 hours. The cumulative distribution of durations, given by $P(t)=\int_{t}^{\infty} p\left(t^{\prime}\right) d t^{\prime}$, was employed to demonstrate the difference between periods of sleep and wake. The periods of time spent in a wake state followed a power-law distribution,

$$
P(t) \sim t^{-\alpha}
$$

where, for the twenty subjects, $\alpha$ had a mean value of 1.3 and a standard deviation of 0.4 . In contrast, the periods of time spent in a sleep state greater than 5 minutes followed an exponential distribution,

$$
P(t) \sim \exp (-t / \tau)
$$

with a characteristic time scale $\tau$. For the twenty subjects investigated, $\tau$ had an average of 20 minutes and a standard deviation of 5 minutes.

A simple model of sleep-wake dynamics uses a latent variable, $x(t)$, to define wake states when $x$ is positive and sleep states when $x$ is negative and satisfies $-\Delta \leq x \leq 0$. The latent variable $x(t)$ is assumed to follow a random walk, which corresponds to the noisy behaviour generated by interactions between competing sleep-active and wakepromoting neurons. To ensure a bias towards sleep, once $x$ moves into the wake state, there is a restoring force to attract it back to the sleep state. The sleep-wake transitions during nocturnal sleep usually demonstrate two features: (i) during short wake periods there is a strong probability of falling asleep and (ii) as the period of wake state is prolonged, the probability of falling asleep is reduced. The model imposes a restoring force that weakens as $x$ moves away from the critical transition value of $x=0$. These observations about nocturnal sleep may be represented by a random walk in a logarithmic potential, $V(x)=b \ln x$, with an associated force $f(x)=-d V(x) / d x=-b / x$, where the bias $b$ gives the magnitude of the restoring force. The model may be written as $x(t+1)=x(t)+\delta x(t)$ with

$$
\delta x(t)= \begin{cases}\varepsilon(t), & \text { if }-\Delta \leq x(t) \leq 0 \quad \text { (sleep) }, \\ -\frac{b}{x}+\varepsilon(t), & \text { if } x(t)>0 \quad \text { (wake) }\end{cases}
$$

where $\varepsilon(t)$ is an uncorrelated normally distributed random variable with zero mean and unit standard deviation. As the distribution of durations spent in the wake state follow the return times of a random walk in a logarithmic potential and therefore provide a power-law distribution for large times with cumulative distribution given by $\alpha=1 / 2+b$. In contrast, the distribution of periods spent in the sleep state is the same as that of return times of a random walk in a space with a reflecting boundary. For large times, the cumulative distribution is exponential with characteristic time $\tau$ satisfying $\tau \sim \Delta^{2}$.

The model parameters, $b$ and $\Delta$ may change during the night to reflect the circadian rhythm. Both $b$ and $\Delta$, were
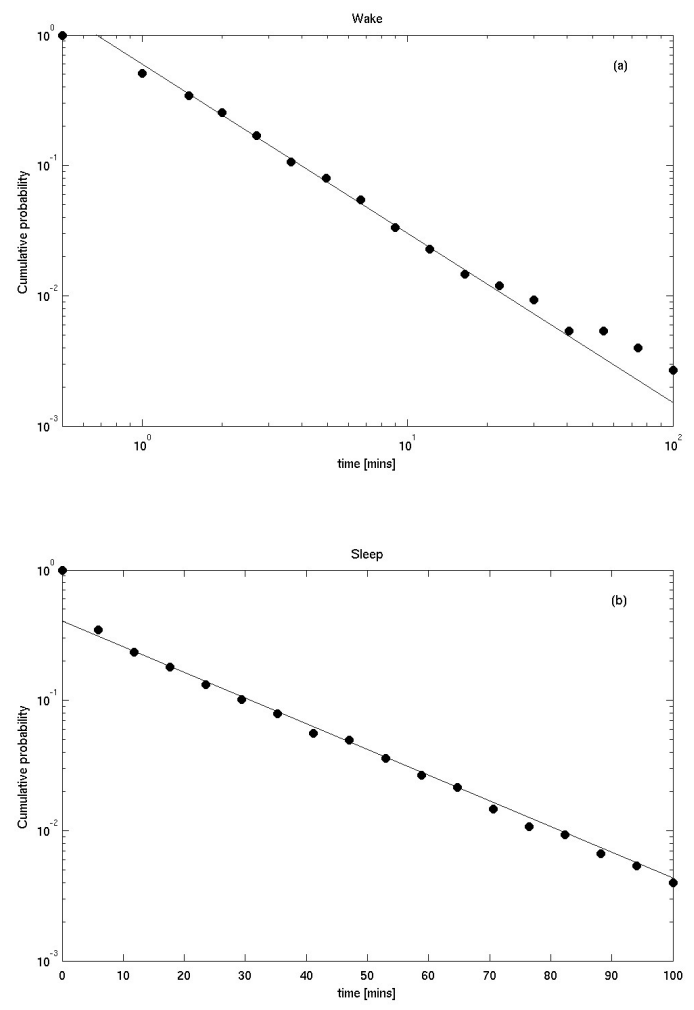

Figure 1. Cumulative distribution functions arising from the dynamics of the sleep-wake model for time duration spent in (a) the wake state (log-log) and (b) the sleep state (log-linear), showing power-law and exponential behaviour respectively.

varied to reproduce the recorded signals and the best estimates suggested that $\Delta$ decreases from $7.9 \pm 0.2$ in the first hours of sleep to $\Delta=5.5 \pm 0.2$ for the final hours of sleep. The bias $b$ was found to remain approximately constant during the night with $b=0.8 \pm 0.1$ [7].

\subsection{Sleep-wake scaling}

The self-similar structure of cardiac interbeat intervals gives rise to an approximate $1 / f$ spectrum, also known as pink noise [4]. In the biomedical literature, this scaling is often quantified using a technique known as detrended fluctuation analysis (DFA) [3]. For a review and comparison of techniques, see [9]. Results from investigations of scaling of during both sleep and wake periods are used to specify suitable scaling coefficients [1]. These results, calculated from 18 healthy subjects (13 females and 5 males with ages between 22 and 71) in the Physionet Normal Sinus Rhythm database [10] gave an average $\beta$ of 1.10 , stan- 
dard deviation of 0.14 , for wake and an average $\beta$ of 0.70 , standard deviation of 0.20 , for sleep.

\subsection{Simulation}

A realisation of a noise process with a particular scaling exponent, $\beta$, may be obtained by (i) computing the discrete Fourier transform (DFT) of a normally distributed white noise with $N$ points, (ii) filtering the resulting Fourier coefficients, $X_{k}$, using $X_{k}^{\prime}=(k / N)^{-\beta / 2} X_{k}$, and (iii) taking the inverse DFT of the filtered Fourier coefficients, $X_{k}^{\prime}$. The new time series were transformed to have means of $\mu_{s}$ and $\mu_{w}$ during sleep and wake where $\mu_{s}$ is normally distributed with mean 1.0 and standard deviation 0.1 and $\mu_{w} / \mu_{s}$ is uniformly distributed on $[0.75,0.85]$. The standard deviations were taken as $5 \%$ of the mean values.

\section{Comparing real and artificial data}

Apart from visual inspection and the reproduction of the dynamics of sleep-wake distribution times, it is also important to evaluate how well the model reproduces empirical heart rate variability statistics. Two common statistics which describe the variability of the RR interval time series over a range of scales are Detrended Fluctuation Analysis (DFA) [11], and Multi-scale Entropy (MSE) [12].

As a representative sample of real RR interval dynamics, we chose the normal sinus rhythm RR interval database [10] which consists of 54 long-term ECG recordings of subjects in normal sinus rhythm ( 30 men, aged 28.5 to 76 , and 24 women, aged 58 to 73). Artificial data was generated using 100 random seeds of the described model, each approximately 24 hours in length. Only the sleep periods of both the real and artificial RR intervals were employed in order to analyse the sleep-wake dynamics.

Figure 3 presents a comparison of the DFA scaling for real and model-generated RR intervals. Note that the model-based RR interval time series has a DFA scaling of $\alpha=1.05$ with a standard deviation of 0.05 over the 100 realisations. The real data exhibits a similar DFA scaling of $\alpha=1.00$ with a standard deviation of 0.06 over the 54 records. Note that the real data tends to display more variance at small values of $n$. This may reflect that the model does not produce enough inter-subject differences for small values of $n$. Both real and artificial populations provide results that are similar to the value of $\alpha=1$ reported in the literature [11].

Figure 4 presents the results for MSE analysis, showing the average sample entropy for both real and artificial data sets. The scale factor gives the number of points averaged to form each element of the coarse-grained time series. Note that both populations have approximately similar values and variances at high scale factors, where any variability over short time scales has been averaged out by
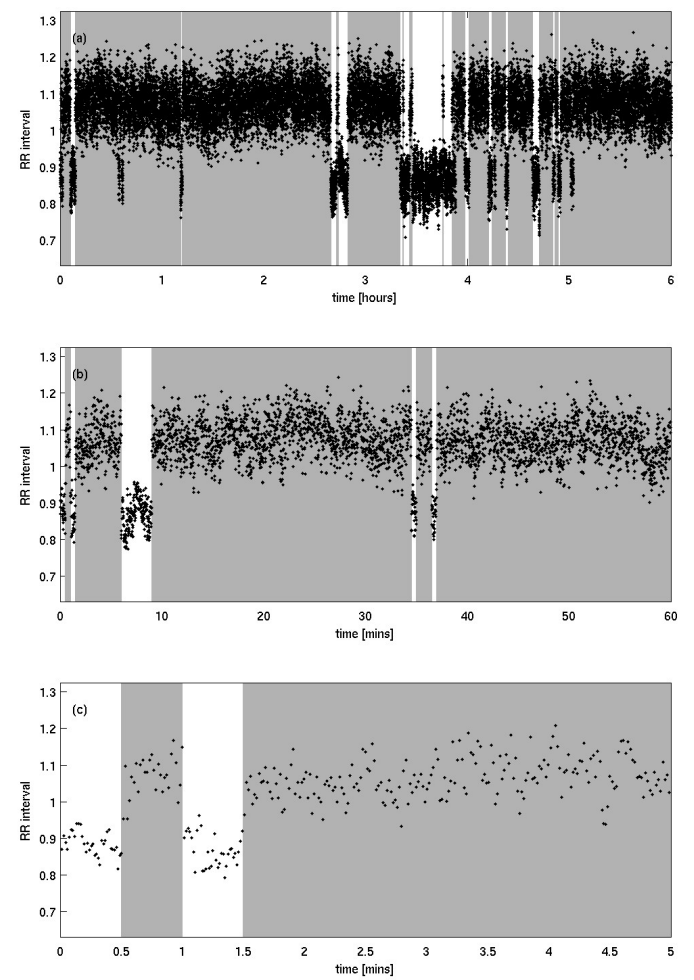

Figure 2. Sleep-wake tachogram simulation showing: (a) first 6 hours, (b) first hour and (c) first 5 minutes. The shaded/white regions indicate state of sleep/wake respectively.

the coarse-graining. In contrast, the sample entropy is underestimated by the artificial RR intervals at the low scale factors. Note that the scale factor of one corresponds to the standard measures of entropy calculated using the raw time series. The level of inter-subject variability is similar for both populations over all scale factors.

The high values of sample entropy at low scale factors for the artificial RR intervals may reflect a lack of structure caused by changes in sympathovagal activity and details of different sleep stages. In addition, the model presented here does not incorporate circadian variability.

\section{Conclusion}

A realistic RR interval generator has been described which preserves both the temporal and spectral properties of RR intervals during periods of sleep and wakefulness. The sleep-wake dynamics were encoded using a binary description of sleep and wake periods. A method of filtering the Fourier coefficients was used to simulate data with spe- 

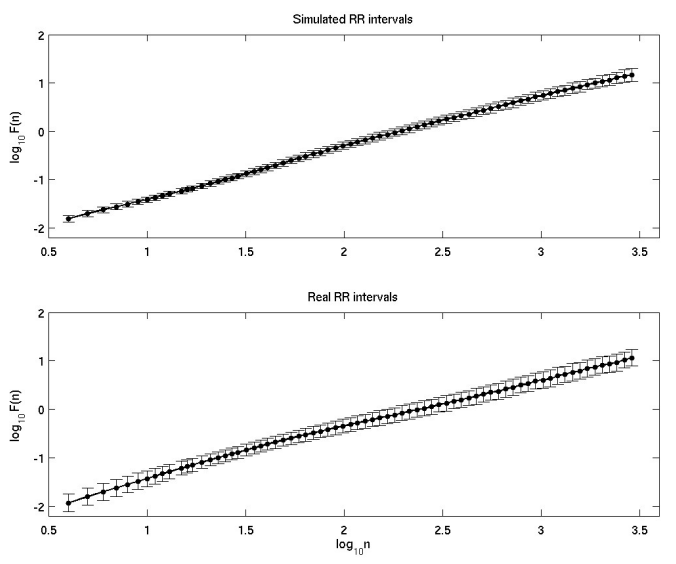

Figure 3. Comparison of mean DFA scaling for 100 artificial and 54 real RR tachograms. Error bars show one standard deviation at each scale.
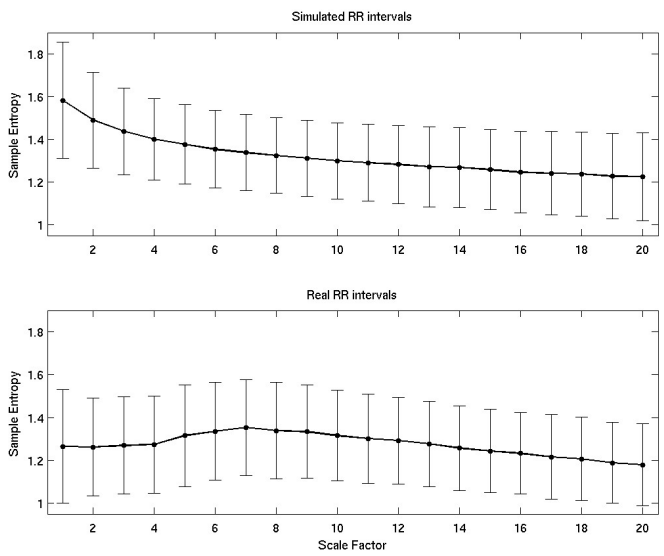

Figure 4. Comparison of mean sample entropy for 100 artificial and 54 real RR tachograms. Error bars show one standard deviation at each scale.

cific scaling properties that reflected the values observed in real RR intervals. The mean, variance and length of each segment mimics empirically observed distributions, including the significant differences observed between periods of sleep and periods of wakefulness. The model's output therefore includes both RR intervals and a binary level of consciousness.

The increasing convergence of the fields of cardiovascular and sleep medicine means that such a model may help researchers develop more robust signal processing algorithms to identify cardiovascular problems both within sleep and during wakefulness.

Further developments of the model could include the introduction of RSA and Mayer waves (see [13]) and sleep staging such that the parasympathetic balance (as observed in the $\mathrm{LF} / \mathrm{HF}$ ratio) varies through sleep stage changes in a manner consistent with physiology.

\section{References}

[1] Ivanov PC, Amaral LAN, Goldberger AL, Havlin S, Rosenblum MG, Struzik ZR, Stanley HE. Multifractality in human heartbeat dynamics. Nature 1999;399:461-465.

[2] Amaral LAN, Goldberger AL, Ivanov PC, Stanley HE. Scale-independent measures and pathologic cardiac dynamics. Phys Rev Lett 1998;81(11):2388-2391.

[3] Peng CK, Hausdorff JM, Goldberger AL. Fractal mechanisms in neural control: Human heartbeat and gait dynamics in health and disease. In Nonlinear Dynamics, SelfOrganization, and Biomedicine. Cambridge, UK: Cambridge University Press, 2000; 66-96.

[4] Kobayashi M, Musha T. 1/f fluctuation of heartbeat period. IEEE Trans Biomed Eng 1982;29:456.

[5] Goldberger AL, Amaral LAN, Hausdorff JM, Ivanov PC, Peng CK, Stanley HE. Fractal dynamics in physiology: alterations with disease and ageing. Proc Natl Acad Sci 2002; 99:2466-2472.

[6] Bunde A, Havlin S, Kantelhardt JW, Penzel T, Peter JH, Voigt K. Correlated and uncorrelated regions in heart-rate fluctuations during sleep. Phys Rev lett 2000;85(17):37363739.

[7] Lo CC, Amaral LAN, Havlin S, Ivanov PC, Penzel T, Peter JH, Stanley HE. Dynamics of sleep-wake transitions during sleep. Europhysics Letters 2002;57(5):625-631.

[8] McSharry PE, Clifford G, Tarassenko L, Smith LA. Method for generating an artificial RR tachogram of a typical healthy human over 24-hours. Computers in Cardiology September 2002;29:225-228.

[9] McSharry PE, Malamud BD. Quantifying self-similarity in cardiac inter-beat interval time series. Computers in Cardiology September 2005;32. In press.

[10] Goldberger AL, Amaral LAN, Glass L, Hausdorff JM, Ivanov PC, Mark RG, Mietus JE, Moody GB, Peng CK, Stanley HE. Physiobank, physiotoolkit, and physionet: Components of a new research resource for complex physiologic signals. Circulations 2000;101(23):e215-e220. URL www. physionet.org.

[11] Peng CK, Havlin S, Stanley HE, Goldberger AL. Quantification of scaling exponents and crossover phenomena in nonstationary heartbeat time series. Chaos 1995;5:82-87.

[12] Costa M, Goldberger AL, Peng CK. Multiscale entropy analysis of complex physiologic time series. Phys Rev Lett 2002;89(6):068102.

[13] McSharry PE, Clifford G, Tarassenko L, Smith LA. A dynamical model for generating synthetic electrocardiogram signals. IEEE Trans Biomed Eng 2003;50(3):289-294.

Address for correspondence:

Patrick E. McSharry

Dept. of Engineering Science / University of Oxford

Parks Road / Oxford OX1 3PJ / UK 
tel./fax: ++44-1865-273095/273905

patrick@mcsharry.net 